\title{
A population-based study of glutathione-S-transferase M1, T1 and P1 genotypes
}

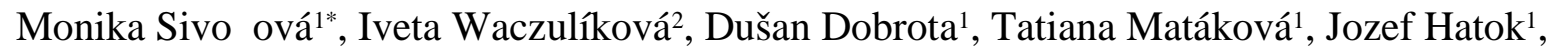
Peter Ra ay ${ }^{1}$ and Ján Kliment ${ }^{3}$

${ }^{1}$ Department of Medical Biochemistry, Comenius University, Jessenius School of Medicine, Malá hora 4, 03601 Martin, Slovakia

${ }^{2}$ Department of Nuclear Physics and Biophysics, Division of Biomedical Physics, Comenius University, Faculty of Mathematics, Physics, and Informatics, Mlynska dolina F1, 84248 Bratislava, Slovakia

${ }^{3}$ Department of Urology, Comenius University, Jessenius School of Medicine and University Hospital, Kollárova 2, 036 59, Martin, Slovakia

*Corresponding author: 
Mgr. Sivo ová Monika, PhD., Department of Medical Biochemistry, Jessenius School of Medicine, Comenius University, Malá hora 4, 03601 Martin, Slovak Republic, tel: +421 43 4131565, fax: +421 43 4136770, email: sivonova@jfmed.uniba.sk

\section{Abstract}

A retrospective study on healthy, unrelated subjects was conducted in order to estimate population glutathione- $S$-transferases (GST) genotype frequencies in Slovak population of men and compare our results with already published data (GSEC project) [1]. A further aim of the study was to evaluate frequencies of the GST polymorphisms also in patients with prostate cancer in order to compare the evaluated proportions with those found in the control subjects. Analysis for the GST gene polymorphisms was performed by PCR and PCR-RFLP. We found that the proportions are not significantly different from those estimated in a European multicentre study or from the results published by another group in Slovakia. We found significantly increased age-standardized prostate cancer prevalence rates in the carriers of GSTM1 null genotype $(\mathrm{P}=0.037)$ and trend for such an increase in the carriers of GSTP1 polymorphism when compared with the respective groups of non-carriers. Because understanding of the contribution of GST gene polymorphisms and their interactions with other relevant factors may improve screening diagnostic assays for prostate cancer, we discuss issues of study feasibility, study design, and statistical power, which should be taken into account in planning further trials.

Key words: glutathine-S-transferase, polymorphism, prostate cancer, slovak population. 


\section{Introduction}

Prostate cancer is the most common cancer among men in industrialized countries with the main risk factor being age over 50. Prostate cancer is uncommon in men less than 45, but becomes more common with advancing age. The average age at the time of diagnosis is 65 [ 2-4]. Since early detection increases survival rate, the prostate-specific antigen (PSA) test and the digital rectal examination should be offered to men annually beginning at age 50. Men at high risk should begin testing at age 45 . The only well-established risk factors for prostate cancer are age, ethnicity, and family history of prostate cancer. However, research in the past few years has shown that genetic, social and environmental factors, particularly diet and lifestyle, likely have an effect as well. It is assumed that increased exposure to procarcinogens and carcinogens contained in tobacco smoke, debris, fermented food, polluted water, air etc., is implicated in multistage carcinogenesis. Therefore assessment of prostate cancer hazard 
from environmental pollution is of increasing importance and inherited differences in the effectiveness of detoxification/activation of carcinogens might play a role in deciding why one man might be at higher risk than another. The GSTM1, GSTT1 and GSTP1 members of the glutathione-S-transferase (GST) multigene family are candidate cancer-predisposing genes, since they are mostly involved in the detoxification of a wide range of environmental and tobacco carcinogens, endogenously produced reactive oxygen species and lipid peroxidation products.

GSTs are phase II enzymes which are responsible for catalyzing the biotransformation of a variety of electrophilic compounds, and have therefore a central role in the detoxification of activated metabolites of procarcinogens produced by phase I reactions [5]. Relation of polymorphisms in these genes to chemical carcinogenesis has been extensively studied in various populations. Several population-based studies have reported prevalence ranging from 47 to $58 \%$ for the GSTM1 deletion genotype and from 13 to $25 \%$ for the GSTT1-null genotype among white Europeans [1,6]. For GSTP1, the found prevalence rates of Ile/Val heterozygosity and Val/Val homozygosity were between (38 - 45.7) \% and (7 to 13) \% respectively [7].

Normal or increased GSTs enzyme activity or levels may protect susceptible tissues from somatic mutation in DNA by facilitating conjugation and subsequent elimination of electrophilic carcinogens. Absent or deficient GST enzyme activity can therefore result in worsened elimination of electrophilic carcinogens, particularly in the presence of very active electrophilic activation by phase I enzymes. GST deficiencies might increase the risk of 
somatic mutation, which subsequently leads to tumor formation [6]. The absence of GSTM1 activity is caused by inheritance of two null alleles (alleles that have a deletion of the GSTM1 gene) similarly; individuals with no GSTT1 activity also have inherited null alleles of the GSTT1 gene. A single nucleotide polymorphism in the GSTP1 gene causes the substitution of isoleucine for valine at amino acid codon 105 (Ile ${ }^{105} \mathrm{Val}$ ) substantially diminishes GSTP1 enzyme activity and lessen effective capacity for detoxification [8,9]. However, the published data about the association of GSTs polymorphism and susceptibility to prostate cancer are controversial. Some studies suggest that the GSTM1, GSTT1 and GSTP1 polymorphisms are associated with prostate cancer susceptibility [10,11], whereas other studies report no association $[12,13]$.

The aims of this study were: 1) to estimate population prevalence of the GSTM1, GSTT1 and GSTP1 gene polymorphisms in Slovak population of men and compare these results with the data published by other groups (GSEC project-Genetic Susceptibility to Environmental Carcinogens); 2) to evaluate frequencies of the GSTT1 and GSTM1 null genotypes and polymorphisms in GSTP1 also in the patients with prostate cancer in order to compare the evaluated proportions with those found in the age-standardized controls; and 3) to examine age-related differences in the frequency distribution of GST polymorphisms in the patients with prostate cancer.

\section{PATIENTS AND METHODS}




\section{Study population}

Blood samples from 228 subjects were obtained from healthy, unrelated subjects living in the north-western part of Slovakia, who were invited to attend the Department of Urology for regular prostate cancer screening between May 2005 and June 2007. The sample (median age of 63, IQR 56-70 years) was used for estimating population GST gene frequencies.

The second part of the study was designed as a case-control study (approximately two controls per one case). A total of 129 prostate cancer patients with histologically verified prostate cancer (median age of 70, IQR 63-74 years) were invited to participate in the project and after signing The informed consent document they were subjected to GST genotyping. The inclusion criteria for the controls were the absence of any previous history of cancer and the serological levels of PSA less than $4 \mathrm{ng} / \mathrm{ml}$.

The present study was performed under the approval of the Ethical Boards of Jessenius School of Medicine, Comenius University.

\section{Chemicals}

Proteinase K was obtained from AppliChem (DE). All the primers, chemicals used for PCR and restriction enzyme were purchased from Eppendorf (USA). All other chemicals used for DNA isolation were purchased from Sigma Co. (USA). 


\section{Genotyping}

Peripheral vein blood was collected in $10 \mathrm{ml}$ heparinized tubes and the specimens were immediately stored at $20^{\circ} \mathrm{C}$ for genotyping. From both, cases and controls, genomic DNA was isolated from peripheral leukocytes by proteinase $\mathrm{K}$ digestion, phenol/chloroform extraction and ethanol precipitation, dissolved in TE buffer $\left(\mathrm{pH} \mathrm{7.5)}\right.$ and stored at $20^{\circ} \mathrm{C}$ until genotype analysis.

A multiplex polymerase chain reaction (PCR) method was used to detect either presence or absence of GSTM1 and GSTT1 genes in the genomic DNA samples simultaneously in the same tube; $\beta$-globin gene was co-amplified and used as an internal control [14]. This technique does not distinguish between heterozygote and homozygote GSTM1- and GSTT1positive genotypes, but it conclusively identifies null genotype [15]. Genomic DNA (100 ng) was amplified in a total volume of $25 \mu \mathrm{l}$ reaction mixture containing 25 pmol of each GST primers (GSTM1: forward 5'-GAA CTC CCT GAA AAG CTA AAG C-3' and reverse 5'GTT GGG CTC AAA TAT ACG GTG G-3'; GSTT1: forward 5'-TTC CTT ACT GGT CCT CAC ATC TC-3' and reverse 5'-TCA CCG GAT CAT GGC CAG CA- 3'); 25 pmol $\beta$-globin gene primers (forward 5'-CAA CTT CAT CCA CGT TCA CC-3' and reverse 5'-GAA GAG CCA AGG ACA GGT AC-3'); $200 \mu \mathrm{mol} / 1$ deoxynucleoside triphosphates; $1 \mathrm{U}$ of Taq polymerase in $10 \times \mathrm{PCR}$ buffer composed of $16.6 \mathrm{mmol} / \mathrm{l}\left(\mathrm{NH}_{4}\right)_{2} \mathrm{SO}_{4}$ and $20.0 \mathrm{mmol} / \mathrm{l} \mathrm{MgCl}, \mathrm{pH} 8.8$. After initial denaturation for $3 \mathrm{~min}$ at $94^{\circ} \mathrm{C}, 39$ cycles were performed for $1 \mathrm{~min}$ at $94^{\circ} \mathrm{C}$ (denaturation), for $1 \mathrm{~min}$ at $60^{\circ} \mathrm{C}$ (annealing) and for $1 \mathrm{~min}$ at $72^{\circ} \mathrm{C}$ (extension), followed by a final step for 5 min at $72^{\circ} \mathrm{C}$. The GSTM1 (215-bp), GSTT1 (480-bp) and $\beta$-globin (268-bp) 
amplified products were visualized by electrophoresis on ethidium-bromide-stained $3 \%$ agarose gel. For deletions of GSTM1 and GST1 no amplified products can be observed, whereas the $\beta$-globin specific fragment confirms the presence of amplifiable DNA in the reaction mixture.

The GSTP1 Ile ${ }^{105}$ Val substitution was detected using the PCR-RFLP approach as the substitution by guanine introduced restriction site that can be recognized by an endonuclease Alw26I. PCR reactions were performed in a total volume $25 \mu$ lof a solution containing $10 \times$ PCR buffer $\left(16.6 \mathrm{mmol} / 1\left(\mathrm{NH}_{4}\right)_{2} \mathrm{SO}_{4}, 20.0 \mathrm{mmol} / 1 \mathrm{MgCl}_{2}, \mathrm{pH}\right.$ 8.8, $1.2 \mu \mathrm{IDMSO}, 1.2 \mu 1$ DTT), $200 \mu \mathrm{mol} / 1$ deoxynucleoside triphosphates, $1 \mathrm{U}$ of Taq DNA polymerase, $100 \mathrm{ng}$ of genomic DNA and 25 pmol of GSTP1 primers (forward 5'-GTA GTT TGC CCA AGG TCA AG-3' and reverse 5'-AGC CAC CTG AGG GGT AAG-3'). The reaction started for 3 min at $94{ }^{\circ} \mathrm{C}$, followed by 5 cycles of PCR (cycle $1: 94^{\circ} \mathrm{C}$ for $15 \mathrm{~s}, 64^{\circ} \mathrm{C}$ for $30 \mathrm{~s}$, and $72^{\circ} \mathrm{C}$ for 1 min) during which the anneling temperature decreased by $1^{\circ} \mathrm{C}$ for each cycle. This step was followed by 30 cycles of denaturation (for $15 \mathrm{~s}$ at $94^{\circ} \mathrm{C}$ ), annealing (for $30 \mathrm{~s}$ at $59{ }^{\circ} \mathrm{C}$ ), and extension (for $1 \mathrm{~min}$ at $72^{\circ} \mathrm{C}$ ). A final polymerization step (for 5 min at $72^{\circ} \mathrm{C}$ ) was carried out to complete the elongation process and yield a 442-bp fragment. A negative control (PCR without template) was included in each set of PCR reactions. Each PCR product (10 $\mu$ l) was digested for 4 hours with restriction enzyme Alw26I (5 U) and electrophoresed on ethidiumbromide-stained $1.5 \%$ agarose gel. The presence of the Ile/Ile allele was detected by 329-, and 113-bp fragments, whereas the Val/Val allele was confirmed by 216-, 113-, and 107-bp 
fragments. The heterozygote Ile/ Val allele was characterized by four fragments consisting of 329, 216, 113 and 107 bp [7].

\section{Statistics}

Age is presented as median and interquartile range (IQR) because the data showed departures from normality (according to Shapiro-Wilk's test). The $\chi^{2}$ method was used to test frequencies of genotypes/allele in prostate cancer patients and controls. The odds ratios (OR), estimates of the relative risk, with $95 \%$ confidence intervals (CI) were computed to assess strengths of association of the genotypes with prostate cancer. All $P$ values cited are twosided alternatives; differences with $P$ values less than 0.05 were judged as statistically significant [16].

\section{Results}

Since previous reports suggest that there are no differences in GSTM1, GSTT1 and GSTP1 allele frequencies by age and sex [17], we conducted a retrospective study on a selected population of men in order to examine whether the gene frequencies were consistent with research findings across Europe. Statistical analysis of data collected from a survey of community sample in the north-western part of Slovakia showed that our estimates were not significantly different from either those found in Caucasian population by Garte and coworkers [1] or those found previously by a research group in Slovakia [1] (Table 1). 
The second purpose of the study was based on suggestion that the GST polymorphism might be associated with increased susceptibility to prostate cancer. Because both the incidence and prevalence of prostate cancer increase rapidly towards the older age, we standardized the genotype data to match the age distribution of the samples. Calculated chi-square for equality of mean column scores and Cramér's V yielded 0.506 and 0.023 , respectively, which did not account for significant differences in the age-standardized GST frequencies between healthy subjects and those diagnosed with prostate cancer. Absence of any association between null genotypes or polymorphism in $G S T$ and prostate cancer was confirmed also by analyzing case-control groups. Table 2 shows the distribution of the GST genotypes among controls and prostate cancer patients. The patients did not have significantly different frequencies in genotypes and alleles in comparison to controls.

Neither the comprehensive score, a pooled value indicating presence at least one variant allele, showed a significantly reduced or unchanged risk of prostate cancer (data not shown). Logistic regression with predictors: age and types of GST enzyme confirmed age as the only risk factor for developing prostate cancer $(\mathrm{P}<0.0001$; OR per one unit change in age amounts to 1.07 (95\% CI 1.04-1.1). Since the age distribution of prostate cancer prevalence first follows a sigmoid-like curve and then gradually declines towards higher age groups, we were interested whether the age distribution curves for GST genotypes followed the same agedependence in the patients with and without defected genes. We found out differences in the age distributions among carriers and non-carriers of GST null genotypes with respect to prostate cancer prevalence. Therefore we conducted an indirect standardization in order to 
adjust these two groups to age. We found significantly increased age-standardized prostate cancer prevalence rates in the carriers of GSTM1 null genotype $(\mathrm{P}=0.037)$ and trend for such an increase in the carriers of GSTP1 polymorphism towards the older age when compared with the respective groups of non-carriers. The differences in the rates for the carriers of GSTT1 null genotype were not significant.

\section{Discussion}

To assess possible association between GST gene polymorphisms and occurrence of prostate cancer in Slovakia, we had to infer from population estimates acquired in the first part of the study on a sample of 228 consecutive men who scheduled appointments in the Department of Urology.

It is known that allele frequencies of the metabolic genes are not equally distributed throughout the human population but follow diverse ethnic and/or geographic-specific patterns. The percentage of individuals who do not express the GSTM1 enzyme due to a homozygous gene deletion is higher in Caucasians and Asians than in Africans. About 20\% of Caucasians, $60 \%$ of Asians and 40\% of Africans do not express the GSTT1 enzyme [1,18]. Our results on GSTMI- and GSTT1-null frequencies, 57\% and 19.7\%, respectively, did not differ significantly either from the values obtained previously by a Slovakian group of researchers $(51.2 \%$ and $18 \%$, respectively) or from those published by other authors [1]. The prevalence rate of Ile/Nal heterozygosity and Val/Val homozygosity was $51.8 \%$ in our control 
subjects. This frequency is also similar to that seen in other studies that analyzed GSTP1 polymorphism [19-21].

Our focusing on the possible link between polymorphism at the GSTP1, GSTM1 and GSTT1 gene loci and susceptibility to prostate cancer stems from the findings that polymorphisms in the genes involved in Phase 1 activation and Phase 2 detoxification of carcinogens can alter prostate cancer susceptibility [22]. It has been suggested that genetic susceptibility may lead to a faster accumulation of DNA damage and to a higher carcinogens accumulation, resulting in an earlier onset of prostate cancer [23]. Some studies have reported a relationship between GST variants and risk of prostate cancer $[9,10,12,13,24]$. Generally, the distribution of genotypes is not related to the stage of disease at the time of diagnosis, tumor histology, or exact site of the tumor. Therefore in our study we assumed there was no significant contribution of these factors to the observed frequencies. Investigation of the GSTP1 gene did not reveal any significant association between heterozygous GSTP1 genotype (Ile/Val) and prostate cancer. However, our results suggest that Val/Val genotype of GSTP1 gene could modulate the risk of prostate cancer, even if this association did not reach statistical significance. We should bear in mind that the inability to reject the null hypothesis could be due to low power of the test because of a relatively small sample size. Therefore, the lack of significance does not necessarily mean equality of the distributions. It is plausible that polymorphism at the GSTP1 locus can play an important role in the susceptibility to different types of cancer. Association of the GSTP1 Val allele with cancer could be expected since the conversion of the amino acid at codon 105 from isoleucine to valine substantially lowers 
activity of the altered enzyme. It has been predicted from molecular modelling that the amino acid at this site lies in a hydrophobic binding site for electrophile substrates and thus affects the substrate binding [23]. On the other hand, there are also studies which did not prove any independent effect of this type of polymorphism on the susceptibility for prostate cancer [2628].

The isoforms GSTM1 and GSTT1 have functional polymorphism in the form of homozygous deletion of either or both genes, which leads to loss of their phenotypic enzyme activities. Individuals vary in their ability to metabolize several DNA-damaging agents because of the polymorphism of these detoxifying enzymes [6]. In the present study, we did not observe significantly different crude rates of the GSTM1 and GSTT1 null genotypes in the men diagnosed with prostate cancer and those in the control group. However, when controlled for age, differences in prevalence of the disease between the carriers and non-carriers of GSTM1 null genotype were evidenced. These findings imply a possibility of earlier onset of the disease in the group of GST null-genotypes carriers. Investigations by the time of diagnosing of prostate cancer are needed to prove the hypothesis of earlier disease-onset in the carriers or at least to provide more scientific data and information.

Our data and data published by other researcher groups suggest that differences in the GST frequencies between prostate cancer patients and control group are relatively small, therefore, it is difficult to separate the groups from each other based on statistical data analysis. Again, high variability in the groups could mask statistical differences due to low power. The easiest way how to improve precision is to increase the number of subjects and patients in the 
experimental design. However, this may not be applicable to all research conditions due to e.g. additional costs, poorer availability of resources, lower population, which compromises the number of subjects eligible for investigation. The possible ways how to achieve a power of at least $80 \%$ are to identify other explanatory variables and control for them, or to apply meta-analysis in order to increase sample size.

Recent studies on GST polymorphism have also evaluated the combined effect of GSTM1 and GSTT1 genotypes, but most of them failed to show any significant association between the joint deficiency of these genes and prostate cancer risk [27,29]; to our knowledge, only one study has reported a significantly increased prevalence of prostate cancer among carriers of both GSTM1 and GSTT1 null genotypes [30]. Some studies, which combined data from other genotypes, have shown that the concurrent lack of GSTM1/GSTT1 and GSTP1 genes posed a significantly increased risk of prostate cancer [21,31,32], however, this has not been proved by other authors [26]. One of the reasons for such discrepancy in the findings might lie in the difficulty of analyzing impact of the modified GST activity on detoxification of known carcinogens. GST has overlapping substrate specificities; therefore deficiency of a single GST isoenzyme may be compensated by the other isoforms. Another important factor is the differential expression of genes for GST in the different cells.

Further, reasons for the inconsistency in results among various studies may be due to differences in the study design, target populations, as well as in the environmental or endogenous exposures. Many have taken for granted that exposure to carcinogens in polluted regions and/or smoking together with gene defect can trigger malignant transformation. 
The variation in published prostate cancer prevalence rates can be attributed partly to methodological differences in survey design, including age distribution of the population surveyed. It is also known that prostate cancer incidence is underestimated, maybe due to poor compliance of elderly with screening recommendations. Thus, regular follow-ups are difficult to achieve and, as a consequence, many men never know they have prostate cancer. It has been reported that calculated prevalence of prostate cancer at death (i.e. histological evidence) for a 60 -year-old man is $32 \%$, but the prevalence in living men (clinically-defined disease) is approximately $4 \%$ [33].

Finally, we will briefly mention two other considerations. Our results on differences in age distribution among carriers and non-carriers of GST null genotypes pointed to an increased prevalence of prostate cancer at younger age. This finding implies a possibility of earlier onset of the disease in the group of GST null-genotypes carriers even if the overall frequencies need not be different in the group of carriers and non-carriers. The second consideration is that, in contrast to the possible role of GST in environmental carcinogenesis, it has been suggested that GST genotypes conferring lower enzyme activity may be of advantage for the patients who are undergoing chemotherapeutic treatment for neoplastic disease because reduced detoxification potentially enhances effectiveness of cytotoxic drugs [ 34]. Although somewhat speculative, the GST polymorphisms might be a protective factor during the period of chemotherapy, as the carriers of GST null genotypes might better respond to the treatment. At present, it is difficult to confidently evaluate GST polymorphisms impact on prostate cancer patients. Apparently, it would be far too simplistic to attribute a complex 
problem such as prostate cancer to any single cause. Even if, methodologically, it is difficult to separate all interfering factors to identify individual changes, there is still a possibility of conducting a carefully designed international and/or multicentric study, or of combining results of several independent studies on the topic.

In conclusion, our results speak for possible association between the GSTP1 Val/Val genotype and occurrence of prostate cancer; however, broad confidence intervals indicate naturally high variability in GST polymorphisms in the population, which has given less weight to the observed differences in GSTP1 Val/Val genotype frequencies between the patients and control subjects

Even if it showed that our study was not designed and powered to detect single gene effects, as well as gene-environment interactions, we cannot exclude that inter-individual differences in GST enzyme activity mediated by polymorphic genes, and reflected in insufficient detoxification of environmental mutagens and carcinogens, may be involved in the pathway, ultimately leading to tumor formation. Because understanding of the contribution of $G S T$ gene polymorphisms and their interactions with other relevant factors may improve screening diagnostic assays for prostate cancer, as well as clinical management of the patients, further studies are desirable to validate observed associations and to identify causal sequence for prostate cancer from $G S T$ gene polymorphisms, providing it exists.

\section{Acknowledgements}


This work was supported by Ministry of Health of the Slovak Republic under the project 2007/45-UK-10 “Genetic polymorphism of xenobiotic metabolising enzymes and susceptibility to prostate cancer in the Slovak population" and by grants UK/264/2006, MVTS Bil/ R/SR/UK/06, AV 4/0013/05, APVT 51-027404 and AV/1106/2004. Authors wish to thank assoc. prof., Ing. O. Križanová, DrSc., and RNDr. B. Sedláková from UMFG SAV Bratislava, Slovakia, for their useful comments and help and to Mrs M. Martin eková and Z. Cetlová for their technical assistance.

\section{References}

[1] Garte S, Gaspari L, Alexandrie AK, et al. Metabolic gene polymorphism frequencies in control populations. Cancer Epidemiol Biomarkers Prev 2001; 10:1239-1248.

[2] Hall WH, Jani AB, Ryu JK, Narayan S, Vijayakumar S. The impact of age and comorbidity on survival outcomes and treatment patterns in prostate cancer. Prostate Cancer Prostatic Dis 2005; 8:22-30.

[3] Tewari A, Johnson ChC, Divine G, et al. Long-term survival probability in men with clinically localized prostate cancer: A case-control, propensity modeling study stratified by race, age, treatment and comorbidities. J Urol 2004; 171:1513-1519. 
[4] Hankey, B, Feuer EJ, Clegg LX, et al. Cancer surveillance series: interpreting trends in prostate cancer-part I: Evidence of the effects of screening in recent prostate cancer incidence, mortality, and survival rates. J Natl Cancer Inst 1999; 91:1017-1024.

[5] Nebert DW, Vasiliou V. Analysis of the glutathione S-transferase (GST) gene family. Hum Genomics 2004; 1:460-464.

[6] Rebbeck TR. Molecular epidemiology of human glutathione S-transferase genotypes GSTM1 and GSTT1 in cancer susceptibility. Cancer Epidemiol Biomarkers Prev 1997; 6:733-743.

[7] Watson MA, Stewart RL, Smith GBJ, Massey TJ, Bell DA. Human glutathione Stransferase P1 polymorphisms. Relationship to lung tissue enzyme activity and population frequency distribution. Carcinogenesis 1998; 19:275-280.

[8] Burim RV, Canalle R, Martinelli AL, Takahashi CS. Polymorphisms in glutathione Stransferases GSTM1, GSTT1 and GSTP1 and cytochromes P450 CYP2E1 and CYP1A1 and susceptibility to cirrhosis or pancreatitis in alcoholics. Mutagenesis 2004; 19:291-298.

[9] Ntais C, Polycarpo A, Ioannidis JP. Association of GSTM1, GSTT1, and GSTP1 gene polymorphisms with the risk of prostate cancer: a meta-analysis. Cancer Epidemiol Biomarkers Prev 2005; 14:176-181.

[10] Debes JD, Yokomizo A, McDonnell SK, et al. Gluthatione-S-transferase P1 polymorphism I105V in familial and sporadic prostate cancer. Cancer Genet Cytogenet $2004 ; 155: 82-86$. 
[11] Komiya Y, Tsukino H, Nakao H, Kuroda Y, Imai H, Katoh T. Human glutathione Stransferase A1, T1, M1, and P1 polymorphisms and susceptibility to prostate cancer in the Japanese population. J Cancer Res Clin Oncol 2005; 131:238-242.

[12] Kidd LC, Woodson K, Taylor PR, Albanes D, Virtamo J, Tangrea JA. Polymorphisms in glutathione-S-transferase genes (GST-M1, GST-T1 and GST-P1) and susceptibility to prostate cancer among male smokers of the ATBC cancer prevention study. Eur $J$ Cancer Prev 2003; 12:317-320.

[13] Medeiros R, Vasconcelos A, Costa S, et al. Metabolic susceptibility genes and prostate cancer risk in a southern European population: the role of glutathione S-transferases GSTM1, GSTM3, and GSTT1 genetic polymorphisms. Prostate 2004; 58:414-420.

[14] Chen H, Sandler DP, Taylor JA, et al. Increased risk for myelodysplastic syndromes in individuals with glutathione transferase theta 1 (GSTT1) gene defect. Lancet 1996; 347:295-297.

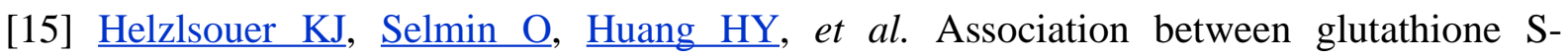
transferase M1, P1, and T1 genetic polymorphisms and development of breast cancer. $J$ Natl Cancer Inst 1998; 90:512-518.

[16] Zar J. Biostatistical analysis, ed. 4. Simon \& Schuster: New Jersey; 2004. Book chapter

[17] Duell EJ, Holly EA, Bracci PM, Liu M, Wiencke JK, Kelsey KT. A population-based, case-control study of polymorphisms in carcinogen-metabolizing genes, smoking, and pancreatic adenocarcinoma risk. J Natl Cancer Inst 2002; 94:297-306. 
[18] Bailey LR, Roodi N, Verrier CS, Yee CJ, Dupont WD, Parl FF. Breast cancer and CYP1A1, GSTM1, and GSTT1 polymorphism: evidence of a lack of association in Caucasians and African Americans. Cancer Res 1998; 58:65-70.

[19] Autrup JL, Thomassen LH, Olsen JH, Wolf H, Autrup H. Glutathione S-transferases as risk factors in prostate cancer. Eur J Cancer Prev 1999; 8:525-532.

[20] Wadelius M, Autrup JL, Stubbins MJ, et al. Polymorphisms in NAT2, CYP2D6, CYP2C19 and GSTP1 and their association with prostate cancer. Pharmacogenetics 1999; 9:333-340.

[21] Steinhoff C, Franke KH, Golka K, et al. Glutathione transferase isozyme genotypes in patients with prostate and bladder carcinoma. Arch Toxicol 2000; 74:521-526.

[22] McIlwain CC, Townsend DM, Tew KD. Glutathione S-transferase polymorphisms: cancer incidence and therapy. Oncogene 2006; 25:1639-1648.

[23] Dalhoff K, Buus Jensen K, Enghusen Poulsen H. Cancer and molecular biomarkers of phase 2. Methods Enzymol 2005; 400:618-627.

[24] Shepard TF, Platz EA, Kantoff PW, et al. No Association between the I105V Polymorphism of the Glutathione $S$-Transferase P1 Gene (GSTP1) and Prostate Cancer Risk: A Prospective Study. Cancer Epidemiology Biomarkers Prev 2000; 9:1267-1268.

[25] Harries LW, Stubbins MJ, Forman D, Howard GC, Wolf CR. Identification of genetic polymorphisms at the glutathione S-transferase $\mathrm{Pi}$ locus and association with 
susceptibility to bladder, testicular and prostate cancer. Carcinogenesis 1997; 18:641644.

[26] Agalliu I, Lin DW, Salinas CA, Feng Z, Stanford JL. Polymorphisms in the glutathione S-transferase M1, T1, and P1 genes and prostate cancer prognosis. Prostate 2006; 66: $1535-1541$.

[27] Gsur A, Haidinger G, Hinteregger S, et al. Polymorphisms of glutathione-S-transferase genes (GSTP1, GSTM1 and GSTT1) and prostate-cancer risk. Int J Cancer 2001; 95:152-155.

[28] Autrup JL, Thomassen LH, Olsen JH, Wolf H, Autrup H. Glutathione S-transferases as risk factors in prostate cancer. Eur J Cancer Prev 1999; 8:525-532.

[29] Katoh T, Yamano Y, Tsuji M, Watanabe M. Genetic polymorphisms of human cytosol glutathione S-transferases and prostate cancer. Pharmacogenomics 2008; 9:93-104.

[30] Srivastava DS, Mandhani A, Mittal B, Mittal RD. Genetic polymorphism of glutathione S-transferase genes (GSTM1, GSTT1 and GSTP1) and susceptibility to prostate cancer in Northern India. BJU Int 2005; 95:170-173.

[31] Kote-Jarai Z, Easton D, Edwards SM, et al. Relationship between glutathione Stransferase M1, P1 and T1 polymorphisms and early onset prostate cancer. Pharmacogenetics 2001; 11:325-330. 
[32] Nakazato H, Suzuki K, Matsui H, et al. Association of genetic polymorphisms of glutathione-S-transferase genes (GSTM1, GSTT1 and GSTP1) with familial prostate cancer risk in a Japanese population. Anticancer Res 2003; 23:2897-2902.

[33] Schröder FH. Screening, early detection, and treatment of prostate cancer: a European view. Urology 1995; 46:62-70.

[34] Willett W. The search for the causes of breast and colon cancer. Nature 1989; 338:389394.

Table 1 Frequencies of GST polymorphisms in the present study, in comparison to those of other studies conducted on Slovak and Caucasian populations.

\begin{tabular}{|c|c|c|c|}
\hline \multicolumn{4}{|c|}{ Genotype frequencies of the GSTP1 gene in Caucasian population and our } \\
\hline & Controls & Caucasians & Probability \\
\hline Ile/Ile & $110 / 228(0.482)$ & $498 / 1137(0.438)$ & 0.22 \\
\hline $\mathrm{Ile} / \mathrm{Val}+\mathrm{Val} / \mathrm{Val}$ & $118 / 228(0.518)$ & $561 / 1137(0.493)$ & 0.51 \\
\hline \multicolumn{4}{|c|}{ Frequency of GSTT1 null genotype in Caucasian population and our controls } \\
\hline & $45 / 228(0.197)$ & $1103 / 5577(0.198)$ & 0.99 \\
\hline \multicolumn{4}{|c|}{ Frequency of GSTM1 null genotype in Caucasian population and our controls } \\
\hline & $130 / 228(0.57)$ & $5583 / 10514(0.531)$ & 0.24 \\
\hline
\end{tabular}




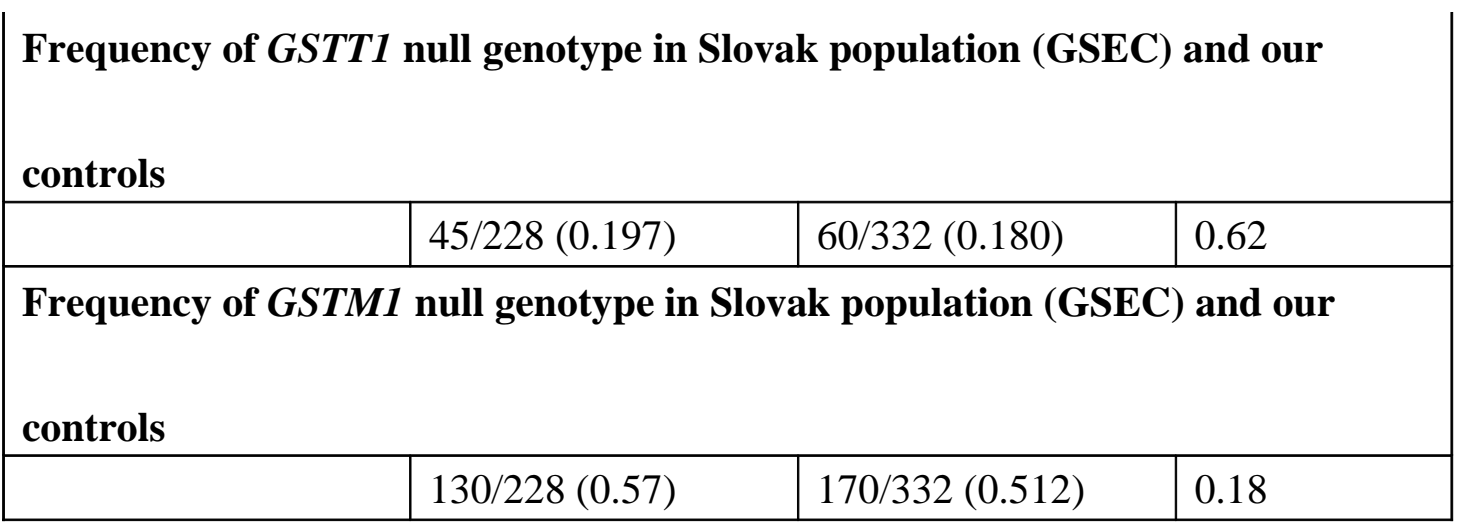

Table 2 Distribution of GST genetic polymorphism in controls and patients with prostate cancer.

Genotype frequencies of the GSTP1 gene in prostate cancer patients (cases) and

\begin{tabular}{|c|c|c|c|c|}
\hline & Controls & Cases & $\begin{array}{l}95 \% \text { CI for } \\
\text { proportion difference }\end{array}$ & Probability \\
\hline Ile/Ile & $103(0.452)$ & $56(0.434)$ & -0.090 to 0.123 & 0.75 \\
\hline Ile/Val & $118(0.517)$ & $67(0.519)$ & -0.109 to 0.104 & 0.97 \\
\hline Val/Val & $7(0.031)$ & $6(0.047)$ & -0.084 to 0.012 & 0.44 \\
\hline $\mathrm{Ile} / \mathrm{Val}+\mathrm{Val} / \mathrm{Val}$ & $125(0.48)$ & $73(0.566)$ & -0.123 to 0.090 & 0.75 \\
\hline \multicolumn{5}{|c|}{ Frequency of GSTT1 null genotype } \\
\hline & $45(0.197)$ & $24(0.186)$ & -0.078 to 0.093 & 0.79 \\
\hline
\end{tabular}


Frequency of GSTM1 null genotype 